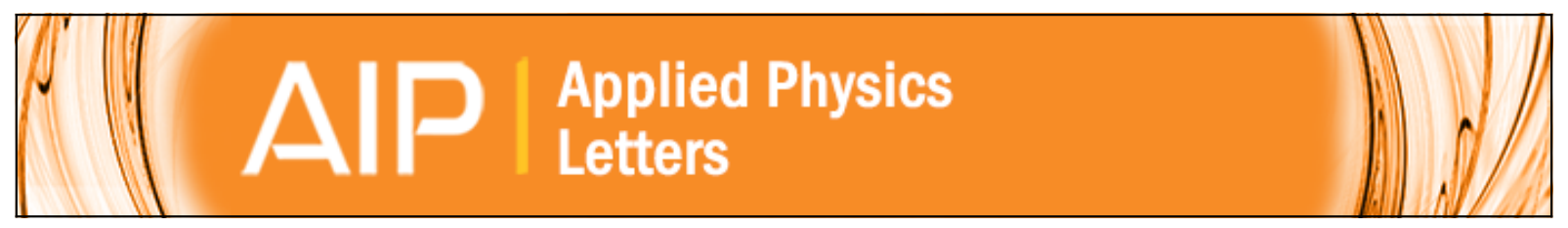

\title{
Amplified spontaneous emission in quaterthiophene single crystals
}

Marco Polo, Andrea Camposeo, Silvia Tavazzi, Luisa Raimondo, Peter Spearman, Antonio Papagni, Roberto

Cingolani, and Dario Pisignano

Citation: Applied Physics Letters 92, 083311 (2008); doi: 10.1063/1.2884687

View online: http://dx.doi.org/10.1063/1.2884687

View Table of Contents: http://scitation.aip.org/content/aip/journal/apl/92/8?ver=pdfcov

Published by the AIP Publishing

\section{Articles you may be interested in}

Enhancement of spontaneous emission rate and reduction in amplified spontaneous emission threshold in electrodeposited three-dimensional $\mathrm{ZnO}$ photonic crystal

Appl. Phys. Lett. 97, 191102 (2010); 10.1063/1.3499274

Photogeneration of charge carrier correlated with amplified spontaneous emission in single crystals of a thiophene/phenylene co-oligomer

J. Chem. Phys. 132, 134509 (2010); 10.1063/1.3367883

Enhanced spontaneous emission rate from single InAs quantum dots in a photonic crystal nanocavity at telecom wavelengths

Appl. Phys. Lett. 91, 123115 (2007); 10.1063/1.2789291

Origin of the amplified spontaneous emission from thiophene/phenylene co-oligomer single crystals: Towards cooligomer lasers

J. Appl. Phys. 99, 013518 (2006); 10.1063/1.2159083

Spontaneous emission from fluorescent molecules embedded in photonic crystals consisting of polystyrene microspheres

Appl. Phys. Lett. 72, 1957 (1998); 10.1063/1.121234 


\title{
Amplified spontaneous emission in quaterthiophene single crystals
}

\author{
Marco Polo, ${ }^{1}$ Andrea Camposeo, ${ }^{1, a)}$ Silvia Tavazzi, ${ }^{2}$ Luisa Raimondo, ${ }^{2}$ Peter Spearman, ${ }^{2}$ \\ Antonio Papagni, ${ }^{2}$ Roberto Cingolani, ${ }^{1}$ and Dario Pisignano ${ }^{1}$ \\ ${ }^{1}$ National Nanotechnology Laboratory of CNR-INFM, Distretto Tecnologico Scuola Superiore ISUFI, \\ Università del Salento, via Arnesano I-73100, Lecce, Italy \\ ${ }^{2}$ Dipartimento Scienza dei Materiali, Università di Milano Bicocca, Via Cozzi 53, I-20125 Milano, Italy
}

(Received 26 November 2007; accepted 31 January 2008; published online 29 February 2008)

\begin{abstract}
The authors demonstrate amplified spontaneous emission (ASE) from quaterthiophene single crystals and study its behavior at low temperature. The room temperature photoluminescence exhibits line narrowing (spectral width down to $10 \mathrm{~nm}$ ) at $553 \mathrm{~nm}$ for excitation fluence larger than $1 \mathrm{~mJ} \mathrm{~cm}^{-2}$. At low temperature $(10 \mathrm{~K})$, ASE is observed from two vibronic transitions at $510 \mathrm{~nm}$ $(0-1)$ and $553 \mathrm{~nm}(0-2)$, with line narrowing down to $5 \AA$, for fluences larger than $100 \mu \mathrm{J} \mathrm{cm}^{-2}$. The stimulated emission cross section is of the order of $10^{-15} \mathrm{~cm}^{2}$, and the ASE is found to be strongly affected by thermally induced dynamic disorder. The emission stability under photopumping is also studied and discussed. (C) 2008 American Institute of Physics. [DOI: 10.1063/1.2884687]
\end{abstract}

Organic crystals are attracting increasing attention as active layer in electronic and optoelectronic devices, such as organic field effect transistors ${ }^{1}$ and solid-state lasers. ${ }^{2}$ Besides their good charge transport properties attaining high carrier mobilities $^{3}$ these materials exhibit a remarkable optical anisotropy induced by the crystalline assembly of their molecules. Indeed, the electromagnetic field within the crystals shows preferential propagation directions, possible selfwaveguiding of the emitted light, ${ }^{4}$ and well defined polarization states, ${ }^{5-7}$ which lead to a high gain coefficient $\left(33 \mathrm{~cm}^{-1}\right)$ in cyano-substituted oligo( $p$-phenylene vinylene) high quality crystals. ${ }^{8}$

The realization of organic crystal-based laser devices may represent a fundamental step toward the achievement of a fully organic laser diode, which requires an in-depth characterization of the optical gain properties of these materials, so far rarely investigated. A widely used approach to probe the optical gain in organic semiconductors consists in investigating their amplified spontaneous emission (ASE) upon excitation by highly intense laser pulses. Some optical transitions are amplified by ASE more than others, leading to a spectrally narrowed emission when the excitation fluence increases. This effect was widely investigated in conjugated polymers and oligomers, ${ }^{9}$ whereas only a few works reported on ASE in organic single crystals, ${ }^{5,6,8,10-19}$ because of the difficulty in growing high-quality and highly pure samples with large active areas and high photoluminescence (PL) quantum yield. Very little is known about the ASE behavior of organic crystals at low temperature $(T)$, which is particularly relevant for assessing the actual potentialities of organic light-emitting semiconductors as active media for laser devices.

In this letter, we report on ASE from quaterthiophene (4T) single crystals and on their gain properties for $T$ ranging from $300 \mathrm{~K}$ to $10 \mathrm{~K}$. Two optical transitions are observed at 510 and $553 \mathrm{~nm}$, occurring from the lowest excited state to the vibronic replicas of the ground state, which show line narrowing (from 6 to $0.5 \mathrm{~nm}$ ) upon increasing the excitation fluence in the range of $70-600 \mu \mathrm{J} \mathrm{cm}^{-2}$. The analysis of the ASE intensity as a

${ }^{a)}$ Electronic mail: andrea.camposeo@unile.it. function of the pumping fluence allow us to estimate the stimulated emission cross section to be as large as $10^{-15} \mathrm{~cm}^{2}$ at $10 \mathrm{~K}$.

As depicted in the inset of Fig. 1(a), 4T is an oligothiophene molecule exhibiting a monoclinic unit cell in the crystal form. 4T was synthesized and purified according to a recently optimized procedure ${ }^{20}$ and single crystals of the low-temperature polymorph (with axis $a=6.085 \AA$, $b=7.858 \AA, c=30.483 \AA, \beta=91.81^{\circ}$, and $Z=4$ ) (Ref. 21) were grown by the floating-drop method. ${ }^{22}$ This technique relies on the dissolution of $4 \mathrm{~T}$ molecules in anisole $\left(10^{-2} \mathrm{M}\right.$ concentration) and the placement of a drop of the obtained
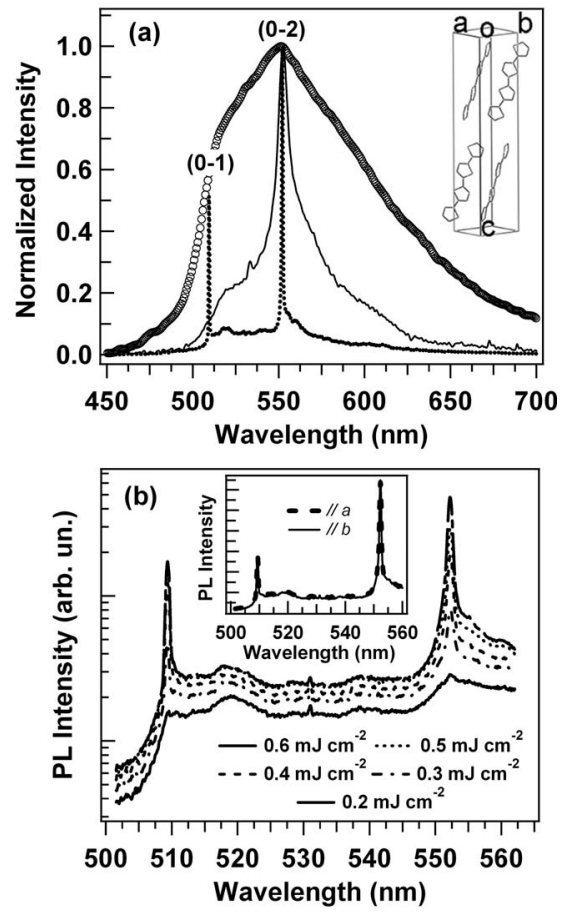

FIG. 1. (a) PL spectra of a $4 \mathrm{~T}$ single crystal at room temperature under excitation fluence of $0.5 \mathrm{~mJ} \mathrm{~cm}$ (circles) and $1.5 \mathrm{~mJ} \mathrm{~cm}^{-2}$ (continuous line) and at $10 \mathrm{~K}$ (dotted line). Excitation fluence for the low-temperature spectrum is $0.5 \mathrm{~mJ} \mathrm{~cm}^{-2}$. Inset: arrangement of the $4 \mathrm{~T}$ molecules in the unit cell. (b) PL spectra acquired at variable excitation fluences at $10 \mathrm{~K}$. Spectra plotted on a log vertical scale for better clarity. Inset: polarized ASE spectra acquired at $10 \mathrm{~K}$ with the analyzer axis parallel to the $a$ crystallographic axis (dashed line) and to the $b$ axis (continuous line). 
solution on the surface of a denser, immiscible, and nonwettable liquid (water in our experiments). The isothermal evaporation of the solvent allows us to obtain crystals with size up to a few centimeters and controlled thickness. The thickness of our samples, measured by a Digital Instrument Dektak 8 profilometer, ranges from few hundreds of nanometers to $1 \mu \mathrm{m}$. The PL spectra were measured pumping the samples by the third harmonic $(355 \mathrm{~nm})$ of a Nd:YAG (yttrium aluminum garnet) microlaser (Alphalas) with a maximum pulse energy of $14 \mu \mathrm{J}$ and a pulse duration of $0.6 \mathrm{~ns}$. The excitation beam was focused on the samples, normally to the substrate, by a quartz lens (spot area $\sim 1 \mathrm{~mm}^{2}$ ). We observed that the intensity of ASE is strongly sensitive to the angle of incidence and polarization of the pumping beam. In fact, the direction of the strongest transition dipole moment lies in the $a c$ plane at an angle $(\alpha)$ with respect to the normal $c^{*}$ axis to the $a b$ face of $27^{\circ}$ (Ref. 4) and, consequently, coupling with the pumping light is maximized at oblique incidence with the polarization of the excitation photons in the $a c$ plane. ${ }^{23}$ The backward PL emission was sent through an optical fiber to a spectrometer (iHR320, Jobin Yvon) equipped with a charge-coupled device camera (Simphony, Jobin Yvon). The measurements were performed for $T$ values ranging from room temperature down to $10 \mathrm{~K}$ by mounting the samples in a He closed-cycle cryostat under vacuum $\left(10^{-4}\right.$ mbar).

Figure 1(a) shows the normalized PL spectra of the crystals at room temperature and at $10 \mathrm{~K}$, respectively. For low excitation fluence, the room temperature emission exhibits a broad and featureless spectrum [full width at half maximum (FWHM) around $100 \mathrm{~nm}$, circles in Fig. 1(a)], whereas it shrinks into a narrow band peaked at $553 \mathrm{~nm}$, of FWHM of about $10 \mathrm{~nm}$ upon increasing $E_{\text {exc }}$ above $1 \mathrm{~mJ} \mathrm{~cm}^{-2}$ [continuous line in Fig. 1(a)]. Instead, the ASE at low temperature [dotted line in Fig. 1(a)] is characterized by the presence of two very sharp peaks (with FWHM about $5 \AA$ ) located at 510 and $553 \mathrm{~nm}$ and corresponding to the (0-1) and (0-2) transitions from the lowest excited state to the vibronic replicas of the ground state (spaced by $184 \mathrm{meV}$ ), respectively. ${ }^{24}$ In fact, the increase of the crystal PL emission upon decreasing $T$ enables ASE also for the (0-1) and (0-2) transitions, which is not observed at room temperature.

The $a$ - and $b$-polarized spectra of the ASE emission acquired at $10 \mathrm{~K}$ are displayed in the inset of Fig. 1(b), clearly showing that the ASE emission from the (0-1) and (0-2) replicas are not polarized. This result is in agreement with the measured polarization of the spontaneous emission peaks (not shown here). Indeed, in contrast to the $b$ polarized $0-0$ $\mathrm{cw}$ line, the replicas are found in both $b$ and $a c \mathrm{cw}$ polarized spectra since all the thermally activated $\mathbf{k}$ states are predicted to contribute. ${ }^{25}$

In order to have a deeper insight into the crystal ASE at low $T$, we investigated the PL spectra at $10 \mathrm{~K}$ at different pumping fluences [Fig. 1(b)]. The (0-2) emission peak is higher than the $(0-1)$, with intensity ratio $\left(I_{0-2} / I_{0-1}\right)$ ranging between 1.5 and 2.0 for excitation fluences between 70 and $600 \mu \mathrm{J} / \mathrm{cm}^{2}$, the specific value of this ratio depending on the sample excited region. This behavior, previously observed in other works, ${ }^{10}$ is due to local variations of the sample thickness and to the distribution of structural defects. At low $T$, both the ASE peaks exhibit a dramatic spectral narrowing down to a FWHM of $5 \AA$, much smaller than the typical linewidths obtained by conjugated polymers. 26
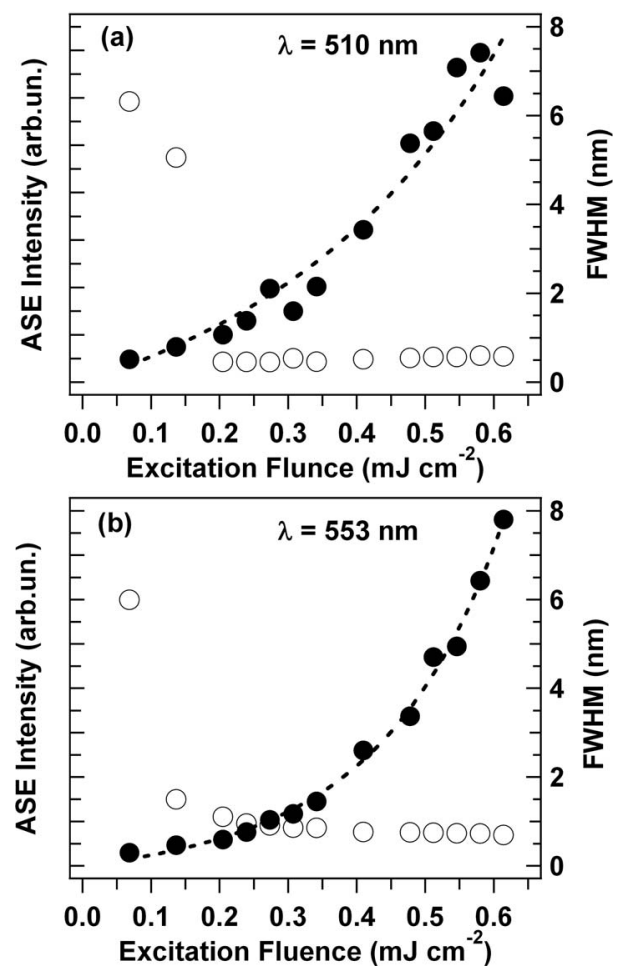

FIG. 2. Dependence of the ASE integrated intensity (solid circles, left scale) and spectral width (FWHM, open circles, right scale) on the pump fluence at $10 \mathrm{~K}$, for the transitions $(0-1)$ at $510 \mathrm{~nm}$ (a) and (0-2) at $553 \mathrm{~nm}$ (b), respectively. The dashed lines are the best fits to the data by Eq. (1).

Figure 2 displays the dependence of the integrated peak intensity (left scale) and of the FWHM (right scale) of the two transitions as a function of $E_{\text {exc }}$. Although ASE is inherently a thresholdless phenomenon, ${ }^{27}$ an experimental threshold is commonly introduced in order to explain the observation of line narrowing above a specific value of $E_{\text {exc }}$, and defined as the halfway fluence between the spontaneous emission and the narrowed ASE linewidths. ${ }^{12}$ We found thresholds of 200 and $100 \mu \mathrm{J} \mathrm{cm}^{-2}$ for the (0-1) and (0-2) transitions, respectively (Fig. 2). In addition, Fig. 2 evidences a nonlinear relationship between the integrated peak intensity $(I)$ and the pump fluence, which is well described by the following exponential-like behavior: ${ }^{27}$

$$
I=A \cdot \frac{(G-1)^{3 / 2}}{(G \ln G)^{1 / 2}} .
$$

In the previous expression, $A$ is a constant that accounts for a Gaussian or Lorentzian lineshape and the optical gain of the amplifier $G$ is given by $e^{\sigma N l}$, where $\sigma$ indicates the stimulated emission cross section, $N$ is the number of excited molecules raised to the upper level, and $l$ is the length of the gain medium. By fitting the experimental data of Fig. 2 by Eq. (1) and assuming a density of excited states equal to the pumping fluence the stimulated emission cross section is estimated to be $4 \times 10^{-15} \mathrm{~cm}^{2}$ for the peak at $553 \mathrm{~nm}$, and 2 $\times 10^{-15} \mathrm{~cm}^{2}$ for the peak at $510 \mathrm{~nm}$. Both these values of $\sigma$ are significantly larger than the typical cross sections $\left(\sim 10^{-16}-10^{-19} \mathrm{~cm}^{2}\right)$ of conjugated materials at room temperature. ${ }^{26,28}$ The ASE of the 4T crystals was investigated in the $T$ range from 10 to $100 \mathrm{~K}$, at a fixed excitation fluence of $500 \mu \mathrm{J} \mathrm{cm}^{-2}$ [Fig. 3(a)]. We found that with decreasing temperature, an increase of the ASE intensity is observed for both transitions due to the suppression of thermally activated migration of excitons toward defect traps ${ }^{24}$ and because of 

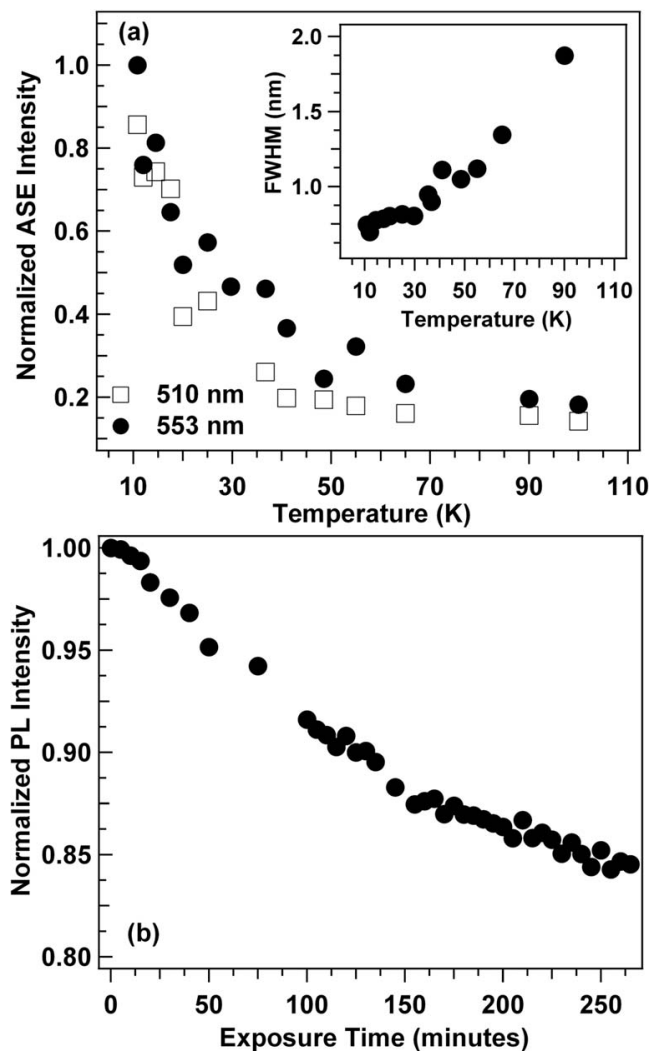

FIG. 3. (a) Dependence of the ASE integrated intensity on temperature, at fixed excitation fluence $\left(E_{\text {exc }}=0.5 \mathrm{~mJ} \mathrm{~cm}^{-2}\right)$ for the transitions (0-1) at $510 \mathrm{~nm}$ (open squares) and (0-2) at $553 \mathrm{~nm}$ (full circles). Inset: (0-2) ASE FWHM vs temperature. (b) Temporal evolution of the integrated PL emission under continuous exposure to the excitation beam (intensity $25 \mathrm{~mW} \mathrm{~cm}^{-2}$ ).

the reduction of the dynamical disorder, ${ }^{29}$ which, in turn, would increase the degree of exciton coherence. We underline that the increase of the ASE intensity is particularly evident below $40 \mathrm{~K}$. At this temperature, indeed, the thermal energy corresponds to the energy of the lattice phonons which have already been demonstrated to be coupled to the lowest exciton state of $4 \mathrm{~T}^{29}$ Therefore, we can attribute the increase of the ASE intensity below $40 \mathrm{~K}$ mainly to the reduction of the dynamic disorder. As far as the peak linewidth is concerned, a monotonous narrowing (FWHM from $2 \mathrm{~nm}$ down to $5 \AA$ ) is observed when $T$ decreases from 100 to $10 \mathrm{~K}$ [inset of Fig. 3(a)]. Also in this case, the behavior is different below and above $40 \mathrm{~K}$. In particular, above $40 \mathrm{~K}$, the FWHM of the ASE peaks decreases linearly upon decreasing $T$, whose trend is expected in a temperature range where static disorder is less effective and the linewidth is mainly affected by the exciton coupling with lattice phonons, in agreement with the previous findings. ${ }^{29}$

Finally, we investigated the stability of the emission from 4T single crystals at room temperature, in air, which is a crucial property for an effective exploitation in light emitting devices. We measured the behavior of the PL intensity under continuous exposure to pumping light, finding a signal decrease by about $15 \%$ in $4 \mathrm{~h}$ [Fig. 3(b)], which constitutes a valuable result compared to the short lifetimes measured in some amorphous polymers. ${ }^{30}$

In conclusion, we demonstrated ASE from 4T organic semiconductor single crystals and investigated its behavior at low temperatures. Two transitions were found to exhibit ASE, with FWHM as low as $5 \AA$ for excitation fluences larger than $100 \mu \mathrm{sm}^{-2}$, and the stimulated emission cross section was estimated of the order of $10^{-15} \mathrm{~cm}^{2}$. We demonstrated that the ASE threshold for $4 \mathrm{~T}$ crystals is reduced by about one order of magnitude by decreasing $T$ down to $10 \mathrm{~K}$. These results indicate oligothiophene crystals as promising active media for organic-based optical amplifiers operating at low temperatures.

This work was supported by the Italian Ministry of University and Research through the PRIN program. ASE measurements on $4 \mathrm{~T}$ were also performed in the framework of the Apulia Explorative Project (PE_086). M. Campione is acknowledged for growing the crystals and L. Silvestri for the helpful discussions.

${ }^{1}$ M. Muccini, Nat. Mater. 5, 605 (2006).

${ }^{2}$ M. Ichikawa, R. Hibino, M. Inoue, T. Haritani, S. Hotta, K. Araki, T. Koyama, and Y. Taniguchi, Adv. Mater. (Weinheim, Ger.) 17, 2073 (2005).

${ }^{3}$ E. Menard, A. Marchenko, V. Podzorov, M. E. Gershenson, D. Fichou, and J. A. Rogers, Adv. Mater. (Weinheim, Ger.) 18, 1552 (2006).

${ }^{4}$ S. Tavazzi, P. Spearman, L. Silvestri, L. Raimondo, A. Camposeo, and D. Pisignano, Org. Electron. 7, 561 (2006).

${ }^{5}$ H. Yanagi, T. Ohara, and T. Morikawa, Adv. Mater. (Weinheim, Ger.) 13, 1452 (2001).

${ }^{6}$ M. Nagawa, R. Hibino, S. Hotta, H. Yanagi, M. Ichikawa, T. Koyama, and Y. Taniguchi, Appl. Phys. Lett. 80, 544 (2002).

${ }^{7}$ S. Tavazzi, A. Borghesi, A. Papagni, P. Spearman, L. Silvestri, A. Yassar, A. Camposeo, M. Polo, and D. Pisignano, Phys. Rev. B 75, 245416 (2007).

${ }^{8}$ W. Xie, Y. Li, F. Li, F. Shen, and Y. Ma, Appl. Phys. Lett. 90, 141110 (2007).

${ }^{9}$ I. D. W. Samuel and G. A. Turnbull, Chem. Rev. (Washington, D.C.) 107, 1272 (2007), and references therein.

${ }^{10}$ D. Fichou, S. Delysse, and J.-M. Nunzi, Adv. Mater. (Weinheim, Ger.) 9, 1178 (1997).

${ }^{11}$ S. Hotta and M. Goto, Adv. Mater. (Weinheim, Ger.) 14, 498 (2002).

${ }^{12}$ M. Ichikawa, R. Hibino, M. Inoue, T. Haritani, S. Hotta, T. Koyama, and Y. Taniguchi, Adv. Mater. (Weinheim, Ger.) 15, 213 (2003).

${ }^{13}$ H. J. Brouwer, V. V. Krsnikov, T. A. Pham, R. E. Gill, P. F. van Hutten, and G. Hadziioannou, Chem. Phys. 227, 65 (1998).

${ }^{14}$ H. Yanagia and T. Morikawa, Appl. Phys. Lett. 75, 187 (1999).

${ }^{15}$ R. Hibino, M. Nagawa, S. Hotta, M. Ichikawa, T. Koyama, and Y. Taniguchi, Adv. Mater. (Weinheim, Ger.) 14, 119 (2002).

${ }^{16}$ X. Zhu, D. Gindre, N. Mercier, P. Frere, and J.-M. Nunzi, Adv. Mater. (Weinheim, Ger.) 15, 906 (2003).

${ }^{17}$ S. Park, O. Kwon, S. Kim, S. Park, M. Choi, N. Cha, S. Y. Park, and D. Jang, J. Am. Chem. Soc. 127, 10070 (2005).

${ }^{18}$ G. Horowitz, F. Kouki, A. E. Kassmi, P. Valat, V. Wintgens, and F. Garnier, Adv. Mater. (Weinheim, Ger.) 11, 234 (1999).

${ }^{19}$ P. A. Losio, C. Hunzicker, and P. Günter, Appl. Phys. Lett. 90, 241103 (2007).

${ }^{20}$ S. Trabattoni, S. Laera, R. Mena, A. Papagni, and A. Sassella, J. Mater. Chem. 14, 171 (2004).

${ }^{21}$ T. Siegrist, C. Kloc, R. A. Laudise, H. E. Katz, and R. C. Haddon, Adv. Mater. (Weinheim, Ger.) 10, 379 (1998).

${ }^{22}$ M. Campione, R. Ruggerone, S. Tavazzi, and M. Moret, J. Mater. Chem. 15, 2437 (2005).

${ }^{23}$ P. Spearman, A. Borghesi, M. Campione, M. Laicini, M. Moret, and S. Tavazzi, J. Phys. Chem. 122, 014706 (2005).

${ }^{24}$ F. Meinardi, M. Cerminara, S. Blumstengel, A. Sassella, A. Borghesi, and R. Tubino, Phys. Rev. B 67, 184205 (2003).

${ }^{25}$ F. C. Spano, J. Chem. Phys. 120, 7643 (2004).

${ }^{26}$ D. Pisignano, E. Mele, L. Persano, G. Paladini, and R. Cingolani, Appl. Phys. Lett. 86, 261104 (2005).

${ }^{27}$ O. Svelto, Principles of Lasers (Plenum, New York, 1989).

${ }^{28}$ M. Anni, G. Gigli, R. Cingolani, M. Zavelani-Rossi, C. Gadermaier, G. Lanzani, G. Barbarella, and L. Favaretto, Appl. Phys. Lett. 78, 2679 (2001).

${ }^{29}$ L. Raimondo, M. Laicini, P. Spearman, S. Tavazzi, and A. Borghesi, J. Chem. Phys. 125, 024702 (2006).

${ }^{30}$ A. Costela, I. Garcia-Moreno, J. M. Figuera, F. Arnat-Guerri, R. Mallavia, M. D. Santa-Maria, and K. Sastre, J. Appl. Phys. 80, 3167 (1996). 